\title{
ORIGINAL ARTICLE Examining factors that contribute to the process of resilience following spinal cord injury
}

\author{
SA Kilic ${ }^{1}$, DS Dorstyn ${ }^{1}$ and NG Guiver ${ }^{2}$
}

Study design: Cross-sectional survey.

Objective: To examine factors that contribute to the process of positive adjustment, or resilience, in an adult community sample with spinal cord injury (SCI).

Setting: South Australian Spinal Cord Injury Service, Hampstead Rehabilitation Centre, South Australia, Australia

Methods: A postal survey comprising standardised measures of resilience (Connor-Davidson Resilience Scale-10 item), self-efficacy (Moorong Self-Efficacy Scale), locus of control (Locus of Control of Behaviour Scale) and psychological distress (Depression Anxiety Stress Scale-21 item).

Results: Of 60 respondents, $58 \%$ reported moderate to high levels of resilience. Resilience correlated significantly with high selfefficacy $(r=0.68, P<0.01)$, internal locus of control $(r=-0.52, P<0.01)$ and low psychological distress (depression $r=-0.68$, $P<0.01$; anxiety $r=-0.55, P<0.01$; stress $r=-0.67, P<0.01)$. In comparison, resilience was not significantly influenced by degree of neuropathic pain $(r=-0.23, P>0.05)$, time since injury $(r=-0.14, P>0.05)$, gender $(t(58)=-0.92, P>0.05)$, lesion completeness $(t(57)=-0.86, P>0.05)$, or SCl diagnosis $(t(58)=-1.21, P>0.05)$. A multiple regression indicated that psychological distress and self-efficacy were the only two variables that uniquely contributed to resilient behaviour.

Conclusion: Resilience is an important psychological process in the longer-term management of SCl which can be promoted by targeting rehabilitation interventions towards mood management in addition to self efficacy beliefs. Larger-scale research will help to validate these results.

Spinal Cord (2013) 51, 553-557; doi:10.1038/sc.2013.25; published online 21 May 2013

Keywords: spinal cord injuries; resilience; self-efficacy; depression; anxiety; rehabilitation

\section{INTRODUCTION}

The process of coping and psychological adjustment to spinal cord injury (SCI) has traditionally focussed on a deficit-based paradigm of mental health by identifying cognitive vulnerabilities, maladaptive behaviours and biomedical risk factors, which lead to poor psychological outcomes, such as depression. ${ }^{1-3}$ This includes the role of negative self-evaluations and coping behaviours in the adjustment process, ${ }^{4}$ the impact of chronic neuropathic pain on psychological outcome $e^{4,5}$ and gender differences in rates of depression post-SCI. ${ }^{3}$

Although researchers have made important contributions towards identifying negative outcomes for individuals with SCI using the deficit-based paradigm ${ }^{2}$ it has not assisted researchers in finding ways to prevent psychological problems from developing., ${ }^{2,6,7}$ In comparison, the strength-based model of mental health, which evolved from the positive psychology movement, focuses on the variables that buffer against mental illness. ${ }^{2,8}$ The strength-based model has also introduced concepts such as 'resilience' in the rehabilitation literature. ${ }^{8}$

Broadly speaking, resilience refers to the personal qualities that enable individuals to positively adjust in the face of a significant adversity, such as a SCI. ${ }^{1,8}$ Researching resilience is important because it enables practitioners to understand why and how up to $54 \%$ of individuals with SCI report a life trajectory characterised by relatively stable and healthy levels of psychological and social functioning. ${ }^{6-8}$ Moreover, the literature emphasises that the personal thoughts and behaviours that contribute to resilience are modifiable and can be improved with psychological treatment. ${ }^{8}$

Despite its relative importance in the psychological adjustment process, the process of resilience post-SCI has been relatively understudied. ${ }^{6,8}$ Furthermore, there are conflicting findings relating to the cognitive, behavioural and injury-specific variables that may offer protection and enhance the adjustment process. ${ }^{8}$ For example, it is argued that the permanent and physical constraints associated with SCI contribute to poor self-efficacy with regard to pain management. ${ }^{4,9}$ Individuals with low pain self-efficacy are more likely to view the ability to cope with their neuropathic pain as unmanageable. ${ }^{4,9}$ In turn, they may catastrophise their pain and/or report higher levels of pain-related disability, which can exacerbate depression ${ }^{4}$ and impede functional independence. ${ }^{5}$ Other studies have found that self-efficacy on its own contributes to a relatively small proportion of the variance in perceived quality of life scores postSCI. ${ }^{10}$ Similarly, there is evidence that internal locus of control is associated with reduced levels of depression and anxiety, ${ }^{11}$ yet it is also argued that individuals with an unrealistic level of self-blame display high levels of psychological distress. ${ }^{12}$ In relation to SCI

${ }^{1}$ School of Psychology, Faculty of Health Sciences, University of Adelaide, Adelaide, South Australia, Australia and ${ }^{2}$ South Australian Spinal Cord Injury Service, Hampstead Rehabilitation Centre, Northfield, South Australia, Australia

Correspondence: Dr DS Dorstyn, School of Psychology, Faculty of Health Sciences, University of Adelaide, Adelaide, South Australia 5000, Australia.

E-mail: diana.dorstyn@adelaide.edu.au

Received 18 January 2013; revised 3 March 2013; accepted 6 March 2013; published online 21 May 2013 
variables, there is suggestion that motor complete injury is significantly associated with higher rates of depression and lower levels of resilience. ${ }^{13}$ However, these latter results were based on a small sample $(n=36)$ and, moreover, have not been replicated.

These mixed findings may partly be explained by differences in the SCI samples examined, with much of the resilience research involving inpatient populations. Consequently, these data may not apply to individuals in the chronic stages of their SCI. Indeed there is some evidence that injured individuals may be at a higher risk of developing poor coping skills, including maladaptive cognitive appraisal, over time, as they experience the ongoing physical and emotional losses associated with their roles within their family, community and society. ${ }^{6,8}$

The following study therefore adds to the SCI literature by examining the biopsychosocial factors that contribute to resilience in an adult community sample. Specific aims were to examine: (1) the relationships between resilience and key variables that may influence longer-term adjustment to SCI-namely cognitive appraisal (that is, self-efficacy, locus of control), psychological distress (that is, depression, anxiety and stress), gender, neuropathic pain, SCI diagnosis, and time since injury; and (2) the degree to which these variables uniquely contribute to the resilience process. Based on the presented literature it was broadly hypothesised that coping styles characterised by high self-efficacy, internal locus of control and low psychological distress, would assist longer-term positive adjustment, whereas psychological distress and degree of neuropathic pain would impede this process. Given the very limited research in this area, the magnitude of these relationships could not be estimated.

\section{MATERIALS AND METHODS}

\section{Sample}

The study population consisted of: (1) adults (that is, aged $\geqslant 18$ years); who had been (2) living in the community with both an acquired and traumatic or non-traumatic SCI; and (3) were admitted to the inpatient Spinal Injuries Unit at Hampstead Rehabilitation Centre, Adelaide, South Australia between the years 2008 and 2011. This included individuals with a newly acquired injury who had completed their primary inpatient rehabilitation in the Unit and those in the chronic stages of SCI who required readmission for management of secondary complications (for example, pressure ulcers, neuropathic pain). Eligible individuals also had to have (4) good English comprehension (that is, at least primary school level education; based on information obtained from medical records); and (5) no cognitive impairment (that is, no diagnosis of traumatic brain injury or dementia; based on information obtained from medical records), which would impact on their ability to provide informed consent for the study.

\section{Measures}

In addition to baseline socio-demographic and injury data, respondents were asked to rate the degree to which they experienced medical comorbidities (for example, neuropathic pain, spasticity) on a Likert Scale of 1-10, with higher scores indicating chronicity. ${ }^{14}$

These data were supplemented with four standardized, self-report measures of psychological functioning. The Connor-Davidson Resilience Scale 10 (CDRISC 10) which measures degree of resilient coping in response to life challenges ${ }^{15}$ Research has shown that the CD-RISC 10 has an acceptable $\alpha$ coefficient of $0.85 .{ }^{15}$ The Depression, Anxiety and Stress Scale (DASS-21), ${ }^{16}$ as a screen of depressive mood, anxiety and stress. The DASS-21 has shown sensitivity in detecting symptoms of depression and anxiety in SCI samples. ${ }^{17}$ The Moorong Self-Efficacy Scale (MSES) ${ }^{9}$ which measures an individual's confidence in performing functional, social, leisure and vocational activities post-SCI. Results have confirmed that the MSES is a valid instrument that is sensitive to real-life changes post-SCI. ${ }^{9}$ Finally, the Locus of Control of Behaviour Scale (LCB), which is ${ }^{11}$ designed to measure the degree of externality or the extent to which individuals perceive that their behaviour and well-being is determined by events beyond their control. The LCB has acceptable internal reliability $(\alpha=0.79)$ and construct validity. ${ }^{11}$ Higher ratings on each of the aforementioned scales indicate stronger endorsement of the measured construct.

\section{Procedures}

Of 209 admissions to the Spinal Injuries Unit, 189 people were eligible for inclusion in this study and were invited to participate. All 189 individuals were mailed an information pack containing a letter of invitation, an information sheet, consent form, the self-report measures and a pre-paid return stamped envelope. A total of 46 completed questionnaires were received from this first mail out. In order to improve this $24 \%$ response rate, a second round of questionnaires was sent to non-responders $(n=143)$ and the study extended for 3 weeks. This resulted in an additional 14 respondents $(n=60)$, equating to a $32 \%$ response rate.

\section{Data analysis}

An a priori power calculation indicated that a sample size of 60 would be sufficient to achieve a statistically significant result $(P<0.05)$ with power set at 0.80 for parametric analyses. ${ }^{18}$ Statistics were calculated using statistical package for the social sciences software (SPSS; Version 19.0, IBM, Armonk NY, USA).

Demographic and injury details of respondents $(n=60)$ were initially compared with that of non respondents $(n=129)$, using independent samples $t$-tests and $\chi^{2}$ analyses. This was to determine whether consenting individuals were representative of the larger group of eligible persons identified for the study.

The association between the primary outcome measure of resilience (CD-RISC 10) and the secondary outcome measures (MSES; LCB; DASS-21; pain; time since injury) was then determined with Pearson's correlation coefficient. Independent samples $t$-tests were also used to examine potential differences in resilience based on gender, SCI type (that is, paraplegia vs quadriplegia), and lesion severity (that is, complete vs incomplete lesion).

Finally, a standard multiple regression was run to examine the individual contribution of the abovementioned independent variables to the dependent variable, resilience (CD-RISC 10). To ensure that the regression data met the assumptions of linearity and normality, scatterplots and collinearity statistics were initially inspected. The Enter method was adopted, whereby all independent variables were entered into the equation simultaneously. This method was considered appropriate because no theoretical predictions had been made concerning the ordering or importance of the variables in relation to resilience. ${ }^{19}$

\section{Statement of Ethics}

Ethic approval for this study was granted by the Royal Adelaide Hospital Research Ethics Committee (protocol no. 120426). Accordingly, all applicable institutional and governmental regulations concerning the ethical use of human volunteers were followed during the course of this research

\section{RESULTS}

\section{Sample comparability}

There was no significant difference between respondents $(n=60)$ and non respondents $(n=129)$ in relation to gender $\left(\chi^{2}(1)=0.44\right.$, $P>0.5$ ) or injury type (paraplegia vs tetraplegia; $\chi^{2}(1)=0.31$, $P>.05$ ), suggesting that the two groups were comparable on these variables. However, there was a significant association between group membership and injury cause $\left(\chi^{2}(1)=6.37, P<.01\right)$, with a higher percentage of traumatic injuries present among respondents $(71 \%$ vs $51 \%)$. Respondents were also significantly older, on average, than SCI peers who did not participate in the survey $(t(189)=2.45, P<.05)$. 


\section{Sample characteristics}

The sample was almost exclusively Caucasian $(n=58 ; 98 \%)$, with only one Hispanic and one Indigenous participant. In terms of injury details (Table 1), both newly acquired and chronic SCIs were represented, with time since injury ranging from $1-37$ years $($ mean $=$ 5.7 years). Similarly, both traumatic and non-traumatic SCIs featured (Table 1). Common causes of traumatic SCI included motor vehicle accident $(20 \%, n=12)$, motorbike accident $(18 \%, n=11)$, or fall $(10 \%, n=6)$. Non-traumatic injuries included neoplastic disorders ( $5 \%, n=3$; cancer metastasis to spine), vascular malformations ( $5 \%$, $n=3$; aneurysms), and inflammatory disorders ( $5 \%, n=3$; transverse myelitis, neuromyelitis optica). Respondents described experiencing multiple medical issues secondary to their SCI, namely spasticity (67\%; $n=40)$, chronic neuropathic pain $(65 \% ; n=39)$, urinary tract infections $(45 \% ; n=27)$, and bowel or bladder dysfunction $(15 \% ; n=9)$.

\section{Table 1 Sample characteristics}

\begin{tabular}{|c|c|}
\hline Demographic and $\mathrm{SCl}$ variables & $\mathrm{N}(\%)$ \\
\hline Age-Mean, (s.d.), range, median & 50.8 (17.0), 19-80, 54 \\
\hline \multicolumn{2}{|l|}{ Gender } \\
\hline Male & $41(68.3)$ \\
\hline Female & $19(31.7)$ \\
\hline \multicolumn{2}{|l|}{ Relationship status ${ }^{a}$} \\
\hline Married & $26(43.3)$ \\
\hline Single & $23(38.3)$ \\
\hline Defacto & $5(8.3)$ \\
\hline Partner living separately & $5(8.3)$ \\
\hline \multicolumn{2}{|l|}{ Education (pre-SCI) ${ }^{a}$} \\
\hline Postgraduate degree & $5(8.3)$ \\
\hline Degree/diploma & $15(25.0)$ \\
\hline High school certificate & $15(25.0)$ \\
\hline Some high school & $16(26.6)$ \\
\hline Apprenticeship/trade certificate & $5(8.3)$ \\
\hline Primary school & $2(3.3)$ \\
\hline \multicolumn{2}{|l|}{ Occupation (post-SCI) } \\
\hline Disability/aged pension & $36(60.0)$ \\
\hline Full-time employment & $12(20.0)$ \\
\hline Part-time/causal employment & $9(15.0)$ \\
\hline Student & $3(5.0)$ \\
\hline Time since injury (in years) $-M$, (s.d.), range, median & 5.7 (7.3), 1-37, 3 \\
\hline \multicolumn{2}{|l|}{ Cause of injury } \\
\hline Traumatic & $42(70.0)$ \\
\hline Non-traumatic & $18(30.0)$ \\
\hline \multicolumn{2}{|l|}{ Lesion $^{a}$} \\
\hline Incomplete & $41(68.3)$ \\
\hline Complete & $18(29.5)$ \\
\hline \multicolumn{2}{|l|}{ Lesion levela } \\
\hline Cervical (C3-C6/7) & $22(36.7)$ \\
\hline Thoracic (T2-T12) & $25(41.7)$ \\
\hline Lumbar (L1-L4) & $4(6.7)$ \\
\hline
\end{tabular}

Abbreviation: $\mathrm{SCl}$, spinal cord injury.

alndicates missing or incomplete data.
Incidence of resilience, self-efficacy, locus of control and psychological distress

The majority $(58 \%, n=35)$ of respondents reported moderate to very high resilience scores (that is, CD-RISC 10 score $\geqslant 30$; Table 2).$^{16}$ On average, respondents also reported a strong sense of self-efficacy (MSES) in terms of managing the consequences of their SCI, and an internal locus of control (LCB). The moderate s.d. associated with the CD-RISC 10, MSES and LCB reflect the small number of outliers detected for these scales, with 10 individuals (17\%) reporting less resilient behaviour (that is, CD-RISC 10 score <20), ${ }^{15}$ low selfefficacy (MSES score <60), ${ }^{10}$ and/or high externality (LCB score $>40){ }^{11}$

In relation to psychological distress (Table 2), most reported subclinical levels of depression $(n=36,60 \%$; score $<10)$, anxiety $(n=53,88 \%$; score $<8)$ and stress $(n=49,82 \%$; score $<15)$, based on the DASS-21 definitions of symptom severity. A small minority ( $n=7 ; 12 \%)$ could be defined as clinical 'cases' having reported severe to extremely severe levels of depression (score $\geqslant 21$ ), anxiety (score $\geqslant 15$ ) or stress (score $\geqslant 26) .{ }^{16}$

\section{Relationships between resilience, psychological, demographic and} SCI variables

As shown in Table 3, there was a significant positive correlation between resilience and self-efficacy $(r=0.68, P<0.01)$, indicating that individuals with more resilient thoughts and behaviours reported higher self-efficacy. In comparison, resilience correlated negatively with

Table 2 Descriptive statistics for each outcome measure

\begin{tabular}{lccc}
\hline Measure & Mean (s.d.) & $\begin{array}{r}\text { Score range } \\
\text { (min-max) }\end{array}$ & $\begin{array}{r}\text { Scale range } \\
\text { (min-max) }\end{array}$ \\
\hline Resilience (CD-RISC 10) & $29.53(7.19)$ & $9-40$ & $0-40$ \\
Self-efficacy (MSES) & $88.19(16.60)$ & $43-112$ & $0-112$ \\
Locus of control (LCB) & $27.55(10.43)$ & $5-48$ & $0-85$ \\
Depression (DASS-21) & $9.03(9.01)$ & $0-38$ & $0-42$ \\
Anxiety (DASS-21) & $6.30(7.22)$ & $0-30$ & $0-42$ \\
Stress (DASS-21) & $8.67(7.38)$ & $0-32$ & $0-42$ \\
Pain (Likert scale) & $4.22(3.39)$ & $0-10$ & $0-10$ \\
\hline
\end{tabular}

Abbreviations: CD-RISC 10, Connor-Davidson Resilience Scale; DASS-21, Depression, Anxiety and Stress Scale; LCB, Locus of Control of Behaviour Scale; MSES, Moorong Self-Efficacy and Stre.

Note: Score range indicates range of scores reported by respondents; Scale range indicates the minimum and maximum scores obtainable per scale.

Table 3 Correlational matrix for the different outcome measures

\begin{tabular}{|c|c|c|c|c|c|c|c|}
\hline Variables & $\begin{array}{c}\text { Self- } \\
\text { efficacy }\end{array}$ & $\begin{array}{c}\text { Locus of } \\
\text { control }\end{array}$ & Depression & Anxiety & Stress & Pain & Time \\
\hline Resilience & $0.68^{* *}$ & $-0.52 * *$ & $-0.68^{* *}$ & $-0.55^{* *}$ & $-0.67 * *$ & -0.23 & -0.14 \\
\hline Self-efficacy & & $-0.56^{* *}$ & $-0.63^{* *}$ & $-0.54^{* *}$ & $-0.58 * *$ & -0.27 & -0.07 \\
\hline $\begin{array}{l}\text { Locus of } \\
\text { control }\end{array}$ & & & $0.46^{* *}$ & $0.46^{* *}$ & $0.49 * *$ & -0.01 & $0.31^{*}$ \\
\hline Depression & & & & $0.69 * *$ & $0.75^{* *}$ & $0.33^{*}$ & -0.10 \\
\hline Anxiety & & & & & $0.79 * *$ & 0.11 & -0.05 \\
\hline Stress & & & & & & 0.21 & -0.05 \\
\hline Pain & & & & & & & -0.20 \\
\hline Time & & & & & & & -0.05 \\
\hline
\end{tabular}


locus of control and general psychological distress (DASS-21 subscales), hence individuals who were less resilient were less inclined to acknowledge personal responsibility for their behaviour and tended to report more symptoms of depression, anxiety and/or stress. Interestingly, the only variable that was significantly related to pain ratings was depression $(r=0.33, P<0.05)$. In addition, there was a significant, albeit small, relationship between locus of control and time since injury $(r=0.31, P<0.05)$, suggesting that individuals in the chronic stages of SCI tended to report greater externality (Table 3 ).

In relation to demographics and SCI diagnosis, independent samples $t$-tests revealed no statistically significant differences in CDRISC 10 scores between males and females $(t(58)=-0.92, P>0.05)$, individuals with paraplegia and those with tetraplegia $(t(58)=-1.21, P>0.05)$, or individuals with a complete versus incomplete SCI lesion $(t(57)=-0.86, P>0.05)$. Hence in this sample, resilience was not significantly influenced by either gender or injury severity (Table 3 ).

\section{Variables contributing to resilience}

Multicollinearity statistics revealed high intercorrelations between the DASS-21 depression, anxiety and stress subscales (that is, $r>0.70$ ), hence these subscales were summed to produce a composite measure of distress. ${ }^{16}$ Five independent variables: self-efficacy, locus of control, general distress, pain and time since injury were therefore included in the final regression model. Initial data screening, based on inspection of normal probability plots and scatterplots, confirmed that the data met the assumptions of linearity and normality. ${ }^{19}$

Results of the multiple regression are shown in Table 4. The combination of these five independent variables accounted for $56 \%$ of the variance in this samples' resilience levels (adjusted $R^{2}=0.56$, $F(5,48)=14.45, P=<0.001)$. Of the five variables, total distress made the largest unique contribution, although self-efficacy also made a statistically significant contribution. The associated narrow confidence intervals indicate that these results were unlikely to have arisen by sampling error. ${ }^{19}$

\section{DISCUSSION}

The purpose of the present study was to expand currently limited knowledge on potential psychological and injury-related contributors to the process of learning to be resilient post-SCI. This was achieved by surveying 60 adults who had lived with their SCI for an average of 6 years. Overall, the sample reported moderate to high levels of resilience, as measured by the CD-RISC 10, in addition to proactive self-appraisals and a normal range of depression, anxiety and stress

Table 4 Standard multiple regression with resilience as the dependent variable

\begin{tabular}{|c|c|c|c|c|c|c|c|}
\hline \multirow[t]{2}{*}{ Predictors } & \multirow[t]{2}{*}{$B$} & \multirow[t]{2}{*}{$S E B$} & \multirow[t]{2}{*}{$\beta$} & \multirow[t]{2}{*}{$t$} & \multirow[t]{2}{*}{$\mathrm{P}$} & \multicolumn{2}{|c|}{$\mathrm{Cl}$} \\
\hline & & & & & & Lower & Upper \\
\hline Constant & 22.78 & 6.84 & & 3.33 & $0.002^{* *}$ & & \\
\hline Self-efficacy & 0.14 & 0.06 & 0.33 & 2.53 & $0.02 *$ & 0.03 & 0.26 \\
\hline Locus of control & -0.03 & 0.09 & -0.05 & -0.39 & 0.69 & -0.21 & 0.14 \\
\hline Total distress & -0.15 & 0.04 & -0.46 & -3.54 & $0.001^{* *}$ & -0.24 & -0.07 \\
\hline Pain & -0.11 & 0.21 & -0.05 & -0.54 & 0.59 & -0.54 & 0.30 \\
\hline Time & -0.15 & 0.10 & -0.15 & -1.46 & 0.15 & -0.35 & 0.05 \\
\hline
\end{tabular}

symptomatology. Furthermore, scores on psychological distress (DASS-21) and self-efficacy (MSES) made a unique and statistically significant contribution to perceived resilience. These results provide partial support for the study's hypotheses in that the process of adjustment was predominately characterised by stable mood and positive self-evaluations.

These results are somewhat consistent with the available literature, which suggests that the majority of individuals adjust successfully to the trauma of a severe and permanent neurological injury, such as a SCI. ${ }^{1,6,8}$ Indeed the present study found a similar incidence (58\%) of at least moderate resilience in a community sample to that reported by inpatient samples with SCI. ${ }^{1,6}$ These findings therefore suggest that psychological adaptation to SCI may prevail in the longer term, despite the challenges associated with community living.

An interesting finding was that chronic pain and SCI severity were not significantly related to the ability to be resilient. This contrasts with previous research which supports a significant association between resilience and physical health. ${ }^{8,13}$ However, degree of neuropathic pain did correlate significantly with depression levels which, in turn, influenced resilience scores. The literature suggests that individuals who report medical comorbidities, such as neuropathic pain, have more difficulty coping with the life challenges that SCI presents and, therefore, are more likely to experience depression. ${ }^{4,5}$

These findings have important practical implications for psychological interventions in SCI rehabilitation. Clinicians should consider both the protective factors (for example, self-efficacy) and risk factors (for example, depression, anxiety) related to resilience in their psychological assessments. ${ }^{8}$ Psychosocial interventions, which promote the cognitive and behavioural characteristics of resilient coping, can then be introduced in the early stages of SCI rehabilitation in order to prevent the onset of future emotional distress. One such program is Coping Effectiveness Training-which has shown to contribute to significant improvements in coping appraisal, rational thinking and problem solving skills. ${ }^{20}$ Similarly, vocational re-training programs have been shown to reinforce positive mood and self-efficacy post-SCI. ${ }^{8}$

Although the findings of this study are interesting, the methodological limitations need to be considered. First, the sample was not completely representative of the broader group of SCI individuals identified as eligible for the study. Those who chose to participate in the study tended to be older and to have sustained their injury by traumatic rather than non-traumatic means. This may have biased the results. It is also possible that individuals with higher degrees of resilience were more likely to participate in the study compared with those with poor coping behaviours, such as amotivation and depression. ${ }^{1,2,8}$ The reported $58 \%$ prevalence estimate of resilience, post-SCI, therefore needs to be interpreted cautiously due to the potentially biased sample.

Second, the small response rate may have likely reduced study power which, in turn, may have increased the probability of a Type 1 error, or the likelihood of detecting significant relationships between the examined variables where there were in fact none. ${ }^{18,19}$ Study power can be improved in future studies by increasing the sample size. It is important to note, however, that this study was undertaken within South Australia's only Spinal Injuries Unit, based at Hampstead Rehabilitation Centre. Consequently, the sample size was constrained by a small clinical population.

Third, although the present study expands upon current knowledge of resilience in SCI outpatient settings, its design is cross-sectional. Therefore, it does not explore whether resilience trajectories of individuals with SCI change over time in response to the ongoing 
stressors of SCI. There is some evidence that resilience remains stable among other injury groups. ${ }^{2,7}$ Further longitudinal research is therefore needed to understand changes in resilience levels among SCI populations over time.

Fourth, the significant findings relating to the relationships between resilience, locus of control and pain need to be interpreted cautiously given that these variables individually contributed to a small proportion of the variance in the final regression model. ${ }^{19}$ Nevertheless, the significant and moderate (56\%) contribution of the combined variables is clinically promising. Further large-scale research would allow more advanced analyses, such as structural equation modelling, to better estimate the causal relationship between these variables. ${ }^{1}$

Finally, the reliance on self-report measures of psychological outcome, which are considered to be subjective, presented a testing bias. ${ }^{14}$ Future SCI research should therefore incorporate clinicianbased ratings, consistent with recommendations for clinical trials. ${ }^{14}$

In summary, the concept of resilience is a growing field in SCI research. The present study adds to this body of knowledge by examining several biopsychological variables that have been considered to offer protection and enhance the resilience process postinjury. The findings suggest that targeted treatment of depression, anxiety and stress symptoms, in combination with interventions that adopt a positive psychology framework may help to maximise longerterm emotional outcomes following SCI. It is anticipated that this preliminary data will, in turn, encourage the development of largerscale research.

\section{DATA ARCHNING}

There were no data to deposit.

\section{CONFLICT OF INTEREST}

The authors declare no conflict of interest.

\section{ACKNOWLEDGEMENTS}

The authors are very grateful for the cooperation of patients and staff at Hampstead Rehabilitation Centre, Northfield, South Australia.
1 Catalano D, Chan F, Wilson L, Chiu C. The buffering effect of resilience on depression among individuals with spinal cord injury: a structural equation model. Rehabil Psychol 2011; 56: 200-211.

2 Quale AJ, Schanke A. Resilience in the face of coping with a severe physical injury: a study of the trajectories of adjustment in a rehabilitation setting. Rehabil Psychol 2010; 55: 12-22.

3 Saunders LL, Krause JS, Focht K. A longitudinal study of depression in survivors of spinal cord injury. Spinal Cord 2012; 50: 72-77.

4 Nicholson Perry K, Nicholas M, Middleton J, Siddall P. Psychological characteristics of people with spinal cord injury-related persisting pain referred to a tertiary pain management centre. J Rehabil Res Dev 2009; 46: 57-67.

5 Krause JS, Brotherton SS, Morrisette DC, Newman SD, Karakostas TE. Does pain interference mediate the relationship of independence in ambulation with depressive symptoms after spinal cord injury? Rehabil Psychol 2007; 52: 162-169.

6 White B, Driver S, Warren AM. Resilience and indicators of adjustment during rehabilitation from a spinal cord injury. Rehabil Psychol 2010; 55: 23-32.

7 de-Roon-Cassini TA, Mancini AD, Rusch MD, Bonanno GA. Psychopathology and resilience following traumatic injury: a latent growth mixture model analysis. Rehabil Psychol 2010; 55: 1-11.

8 Craig A. Resilience in people with physical disabilities. In Kennedy P (Ed.) The Oxford Handbook of Rehabilitation Psychology. Oxford University Press: Oxford, p 474-491, 2012.

9 Middleton JW, Tate RL, Geraghty TJ. Self-efficacy and spinal cord injury: psychometric properties of a new scale. Rehabil Psychol 2003; 48: 281-288.

10 Middleton JW, Craig A, Tran Y. Relationship between quality of life and self-efficacy in persons with spinal cord injuries. Arch Phys Med Rehab 2007; 88: 1643-1648.

11 Craig A, Franklin J, Andrews G. A scale to measure locus of control of behaviour. $\mathrm{Br} \mathrm{J}$ Med Psychol 1984; 57: 173-180.

12 Chung MC, Preveza E, Papandreou K, Prevezas N. The relationship between posttraumatic stress disorder following spinal cord injury and locus of control. J Affect Disord 2006; 93: 229-232.

13 Shin JC, Goo HR, Yu SJ, Km DH, Yoon SY. Depression and quality of life in patients within the first 6 months after spinal cord injury. Ann Rehabil Med 2012; 36: 119-1.

14 Bryce TN, Budh CN, Cardenas DD, Dijkers M, Felix ER, Finnerup NB et al. Pain after spinal cord injury: an evidence-based review for clinical practice and research. J Spinal Cord Med 2007; 30: 421-440.

15 Campbell-Sills L, Stein MB. Psychometric analysis and refinement of the Connor Davidson Resilience Scale (CD-RISC): validation of a 10-Item measure of resilience. $J$ Trauma Stress 2007; 20: 1019-1028.

16 Lovibond SH, Lovibond PF. Manual for the Depression Anxiety Stress Scales, 2nd edn. Psychology Foundation: Sydney, 1995.

17 Mitchell MC, Burns NR, Dorstyn DS. Screening for depression and anxiety in spinal cord injury with DASS-21. Spinal Cord 2008; 46: 547-551.

18 Cohen J. A power primer. Psychol Bull 1992; 112: 155-159.

19 Field A. Discovering Statistics Using SPSS, 3rd edn. Sage: London, UK, 2009

20 Kennedy P, Duff J, Evans M, Beedie A. Coping effectiveness training reduces depression and anxiety following traumatic spinal cord injuries. $\mathrm{Br} J$ Clin Psychol 2003; 42: 41-52. 